\title{
Is the Atlantic Multidecadal Oscillation (AMO) a statistical phantom?
}

\author{
M. Vincze and I. M. Jánosi \\ Department of Physics of Complex Systems, Eötvös Loránd University, Pázmány P. s. 1/A, 1117 Budapest, Hungary
}

Received: 10 January 2011 - Revised: 16 June 2011 - Accepted: 5 July 2011 - Published: 14 July 2011

\begin{abstract}
In this work we critically compare the consequences of two assumptions on the physical nature of the AMO index signal. First, we show that the widely used approach based on red noise statistics cannot fully reproduce the empirical correlation properties of the record. Second, we consider a process of long range power-law correlations and demonstrate its better fit to the AMO signal. We show that in the latter case, the multidecadal oscillatory mode of the smoothed AMO index with an assigned period length of 50-70 years can be a simple statistical artifact, a consequence of limited record length. In this respect, a better term to describe the observed fluctuations of a smooth power-law spectrum is Atlantic Multidecadal Variability (AMV).
\end{abstract}

\section{Introduction}

The title of this work is adopted from a remarkable article by Godfrey et al. (2002), where the authors pointed out that mere sampling effects perfectly explain a famous weather folklore (January Thaw), which is an illusory regular warm deviation from the annual cycle during late January in the northeastern US. A more direct motivation of our analysis is provided by Thompson et al. (2010), who have reported on a rapid drop in Northern Hemisphere sea surface temperatures (SST) around 1970. The timescale of the observed drop is much shorter than changes in tropospheric aerosol loadings or slow internal variability such as the Atlantic Multidecadal Oscillation (AMO) index, challenging previous attempts to explain global patterns of 20th century climate variables. Thompson et al. (2010) argue that filtering out high frequency components from a signal can lead to information loss about existing physical processes of relatively

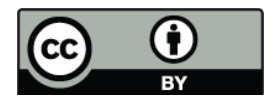

Correspondence to: I. M. Jánosi

(janosi@ lecso.elte.hu) short characteristic times, thus easily masking e.g. jumpwise changes. Fluctuations of mean SST on monthly timescales are usually considered as "pure noise" which has nothing to do with oceanic dynamics, therefore only "slow enough" (like AMO) modes are respected as physical signals.

There is a vast literature regarding the question whether a climatic time series is in fact a result of a pure deterministic process with some characteristic frequency or a stochastic process which exhibits "apparent" periodicity (see e.g. Knight, 2009, and references therein). Best known examples are probably the North-Atlantic Oscillations (NAO) (Hurrell, 1995), the Southern Oscillation Index (SOI) (Trenberth, 1984; Cane and Zebiak, 1985), the Dansgaard-Oeschger events (Ditlevsen et al., 2005) or the glacial-interglacial oscillations that most theories have tried to link to the Milankovitch forcing while others suggested underlying stochastic mechanisms (Ganopolski and Rahmstorf, 2002; Ashkenazy and Tziperman, 2004; Huybers and Wunsch, 2005).

Low frequency oscillations of cool and warm phases in sea surface temperatures in the North Atlantic basin have been identified in instrumental data since 1856 (Kushnir, 1994; Schlesinger and Ramankutty, 1994; Sutton and Allen, 1997; Kerr, 2000; Enfield et al., 2001; Goldenberg et al., 2001) and in proxy data for centuries (Gray et al., 2004). The term Atlantic Multidecadal Oscillation (AMO) was coined by Kerr (2000). The AMO index is introduced by Enfield et al. (2001) as a ten years running mean of monthly SST anomalies, averaged over the Atlantic basin, north of the Equator. The smoothed time series (shown in Fig. 1) exhibits cooler than average SST values in the periods 1900-1925 and 19651995 with warmer periods at the end of the nineteenth century, during 1925-1965, and in the last decade. Note that a global linear trend is removed from the original monthly time series, however it is so weak (the mean SST warming slope is $2.16 \times 10^{-3} \mathrm{~K} \mathrm{yr}^{-1}$ ) that it makes no difference in the following analysis. The relative shortness of the instrumental

Published by Copernicus Publications on behalf of the European Geosciences Union and the American Geophysical Union. 


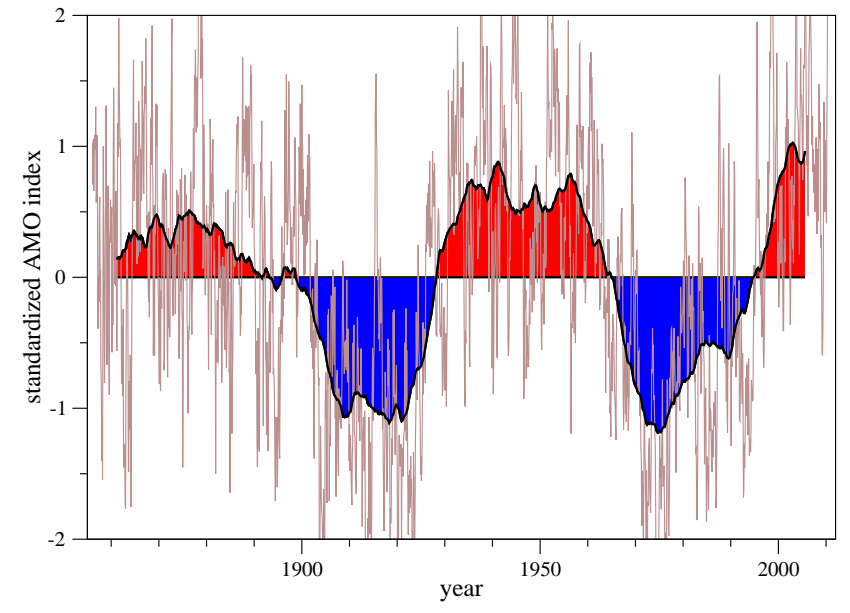

Fig. 1. Standardised monthly mean SST anomalies (thin line) and AMO index (ten years running mean, thick line and red/blue colours) calculated from the Kaplan SST data set which is updated monthly at http://www.cdc.noaa.gov/Timeseries/AMO/.

climate record compared to an assumed period length of 50 70 years, however, limits confidence of clearly establishing a real oscillatory mode.

This impression is further strengthened by comparing the instrumental signal with the tree ring proxy record by Gray et al. (2004) in Fig. 2. The reconstructed annual mean SST anomaly, and its ten years running mean (proxy AMO) have a somewhat limited overlap with the instrumental signals in the appropriate time interval, nevertheless the proxy AMO lacks the signature of a more or less stable oscillatory mode.

An alternative definition of AMO index was proposed by Trenberth and Shea (2006). The main difference is that they computed mean SST values for the world ocean and determined the difference between this "background" and the North-Atlantic average. This modified AMO index has a decreased variability, however the "warm" and "cold" phases are almost overlap with the signal shown in Fig. 1. Other modifications, effects of different detrending and background removal procedures and problems with the signal interpretations are summarized in details by Knight (2009).

Numerical models have a distinguished role to simulate much longer periods than covered by reliable measurements (Frankignoul and Hasselmann, 1977; Delworth et al., 1993; Timmermann et al., 1998; Dong and Sutton, 2005; Jungclaus et al., 2005; Knight et al., 2005; Frankcombe et al., 2009; Knight, 2009; Ottera et al., 2010). The key element common in all models is a link between the AMO and the Atlantic meridional overturning circulation, however characteristic time scales of the variability are not satisfactorily explained. A recent numerical work by Park and Latif (2010) has produced an AMO signal over a simulated interval of 1000 years, and multidecadal oscillations of a characteristic period about 60 years have identified. Note, however, that this result is obtained by band-pass filtering of the original SST time series in the period range 30-90 years, and the authors have consistently used the term Atlantic Multidecadal Variability (AMV) instead of AMO throughout the paper (Park and Latif, 2010). The picture is further complicated by observations of variability on 20-30 year time scales of sub-surface temperature (Frankcombe et al., 2008), and tide gauge records (Frankcombe and Dijkstra, 2009) in the North Atlantic.

Here we propose that the mean SST anomaly signals exhibit long range power-law correlations, instead of being a simple low-order autoregressive process. (Long range correlations for local SST values are detected already by e.g. Monetti et al. (2003).) As a consequence, the apparent multidecadal oscillation represented by the AMO index can be explained as a simple finite size effect. We do not question the variability of mean SST anomalies on timescales of decades, however we intend to refine the picture by demonstrating the probable lack of a fixed characteristic frequency. This finding can resolve the many controversial estimates on oscillatory time scales in simulations and proxy reconstructions.

\section{Correlation properties}

In order to compare measured and artificial model time series $x(t)$, we always perform the usual standardisation by the empirical mean value $\langle x\rangle$ and standard deviation $\sigma=$ $\sqrt{\left\langle x^{2}\right\rangle-\langle x\rangle^{2}}$ as $X(t)=[x(t)-\langle x\rangle] / \sigma$, as in Figs. 1 and 2. We will return to the importance of this step at the particular tests.

Since the partial autocorrelation function of the standardised monthly mean SST anomaly $I_{n}$ (Fig. 1) drops to zero in a single step (not shown here), moving average (MA) processes cannot come into question (von Storch and Zwiers, 1999). Fits of autoregressive AR $(m)$ models with increasing orders $m$ do not results in a significant improvement compared to the simplest first order AR(1) hypothesis:

$I_{n+1}=a_{1} I_{n}+\xi_{n}$,

where $a_{1}=0.9034684$, and $\xi_{n}$ is a random variable drawn from a Gaussian IID ensemble of standard deviation $\sigma_{\xi}=$ $\sigma_{I} \sqrt{1-a_{1}^{2}}=0.428654$ (note that $\sigma_{I} \equiv 1$ as a consequence of standardisation). As a measure of goodness of fit, we list the square-root mean error (based on observed value minus onestep-ahead forecast) for $\operatorname{AR}(m)$ fits with $m=1 \ldots 5: 0.4310$, $0.4311,0.4306,0.4301,0.4294$. Even at $m=20$, the mean forecast error remains 0.4247 , the improvement is negligible.

As a next step, we produced an artificial series of 185500 data points by iterating Eq. (1) with the fitted parameters, and split into 100 pieces of equal length of the original monthly mean SST anomaly series. The scatter plot of the empirical mean value and standard deviation for each individual piece is shown in Fig. 3 with black circles. As expected, the splitting resulted in some statistical shifts at the short segments, 


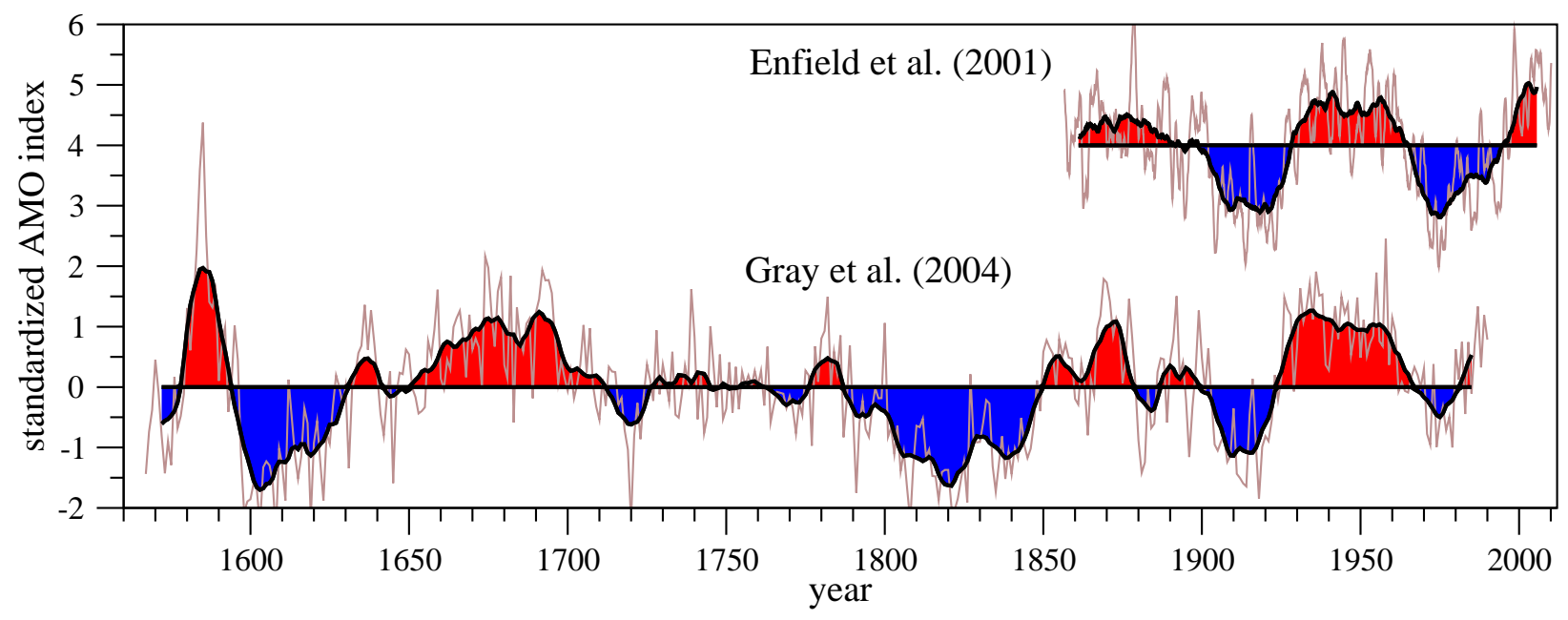

Fig. 2. Top: standardised annual mean SST anomaly and AMO series as in Fig. 1, shifted upward for a clear visualisation. Bottom: standardised annual mean SST anomaly (thin line) and its ten years running mean (thick line, red and blue coloured) determined from the tree ring proxy data set by Gray et al. (2004) ftp://ftp.ncdc.noaa.gov/pub/data/paleo/.

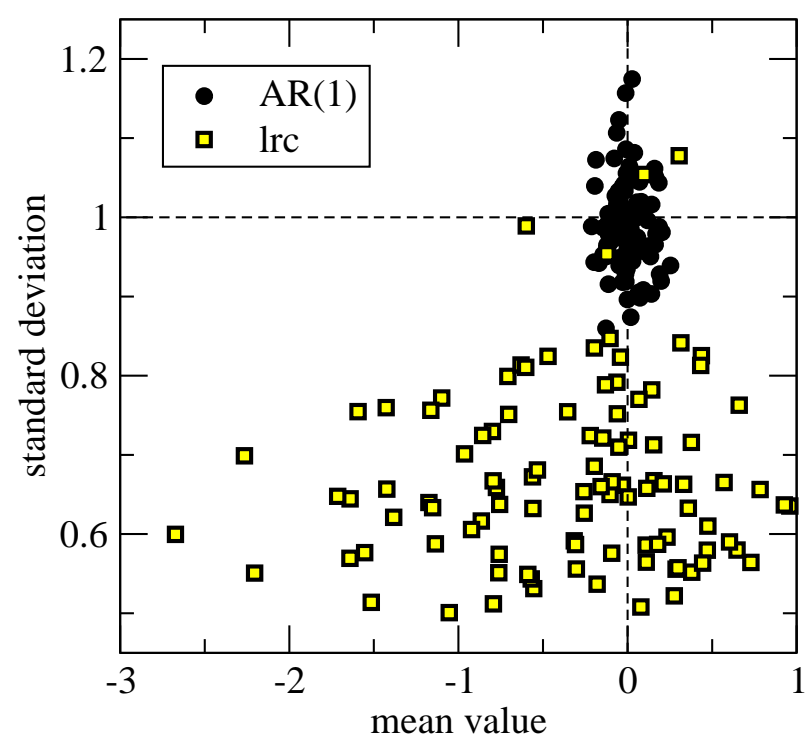

Fig. 3. Scatter plot of the individual mean values and standard deviations for two sets of model time series. The first series is produced by the AR(1) model Eq. (1) with the parameters fitted to the monthly SST signal. The second long-range correlated (lrc) signal of spectral exponent $\beta=0.6$ is produced with the inverse Fourier method, see e.g. Fox (1987). Both time series of 185500 data points are split into 100 equal pieces, and the individual means and standard deviations are plotted (see legends).

therefore standardisation was performed separately with the individual mean values and standard deviations prior to subsequent analysis.
Temporal correlation properties were evaluated by two methods: detrended fluctuation analysis (DFA) and Fourier transform (power spectrum). Both of them are considered nowadays as standard procedures, therefore we skip technical details ("Google Scholar" gives almost four thousand hits for searching DFA). Here we emphasise only the key aspect that in the mathematical sense these methods (together with the computation of autocorrelation function) provide the very same information about two-point temporal correlations. For example, a long range correlated (lrc) process by definition obeys a power-law decaying autocorrelation function $A(\tau)=\langle X(t+\tau) X(t)\rangle \sim \tau^{-\alpha}$ with an exponent $0<\alpha<1$. Simultaneously, its power spectrum has a similar form $S(f) \sim f^{-\beta}$, and the DFA $p$ fluctuation function is also power-law: $F_{p}(w) \sim w^{\delta}$. Furthermore, the exponents obey cross-relations (Heneghan and McDarby, 2000), e.g.:

$\alpha=2(1-\delta), \quad \beta=2 \delta-1, \quad \alpha+\beta=1$.

The mathematical equivalence does not mean that these methods are equally efficient for finite (and often noisy) data, e.g. the Fourier transformation usually provides more robust result than a direct computation of the autocorrelation function. The DFA $p$ procedure has a benefit that it removes a global trend [a polynomial of order $(p-1)$ ] from the original series, thus it can handle nonstationarities.

For the very reason, we determined DFA $p$ fluctuation functions with $p=2 \ldots 5$ for the standardised monthly SST anomaly shown in Fig. 1 and for each AR(1) model sequence separately. Since we did not observe differences for increasing $p$ values, we show only the results of DFA2 computations in Fig. 4a. For increasing time window sizes $w$ (in units of month), the statistical inaccuracies result in a widening band for the model sequences, nevertheless their behaviour follows the expectations (Király and Jánosi, 2002). For short 


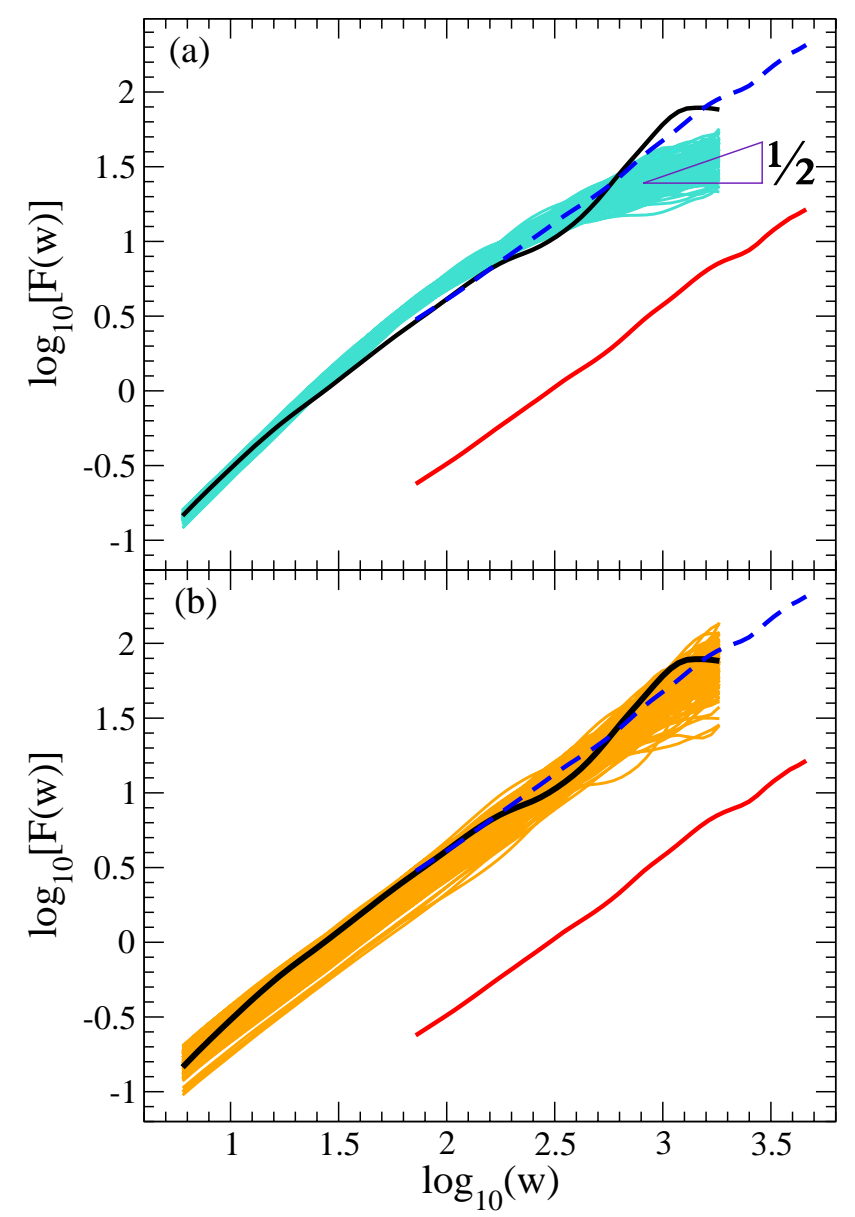

Fig. 4. The logarithm of DFA2 fluctuations $\log _{10}[F(w)]$ as a function of the logarithm of sliding window size $\log _{10}(w)$ for the model sequences (thin coloured lines) and for the monthly mean SST anomaly (thick black curve). The thick red line is the same for the tree ring proxy annual mean SST (see Fig. 2), also shown as a blue dashed line shifted upward. (a) Fitted AR(1) model series, and (b) long range correlated series with $\beta=0.6$.

times an AR(1) process has strong "memory" indicated by a large slope of DFA curves, but this slope gradually decreases to the asymptotic value of $1 / 2$. The empirical monthly SST sequence does not really fit into this band: the AR(1) model systematically overestimates observed correlations for the intermediate times [40 - 135] months, and definitely underestimates them over 690 months (note the $\log _{10}$ scales in Fig. 4).

The limited length of the monthly SST anomaly series (1855 data points in our analysis) and observational noise unavoidably yield to statistical uncertainties, nevertheless the black DFA curve in Fig. 4a seems to be much more linear than the cyan AR(1) model curves. Therefore it seems plausible to test the assumption of long range power-law correlations (which would result in a straight line in Fig. 4a). Power-law correlated model series can be easily produced e.g. by the classical inverse-Fourier method, where the input

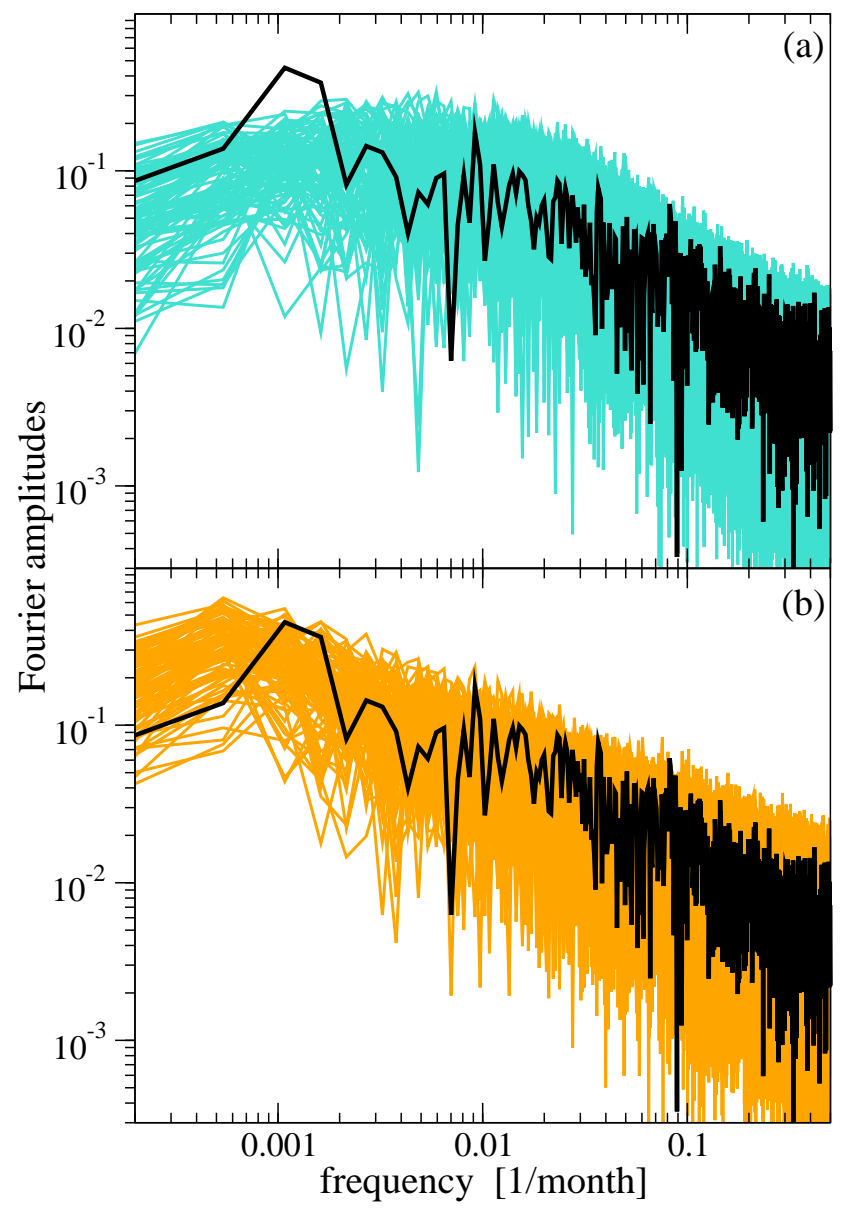

Fig. 5. Power spectra of (a) fitted AR(1) and (b) lrc model series ( $\beta=0.6$ ) (thin coloured lines), together with the spectrum for the monthly mean SST anomaly record (thick black line). Note the double-logarithmic scales. (In each case, the standard Welch windowing was applied prior to the Fourier transform.

is a prescribed spectrum with a given $\beta$ and random phases, and the output is a scalar time series (Fox, 1987). (Some care should be taken to handle continuation upto the zero frequency, but the appropriate methods are also widely discussed in the literature.) The main difference between a long range correlated (lrc) and AR(1) series is clearly illustrated in Fig. 3 (yellow squares): the individual means and standard deviations cover a much larger range when a long standardised lrc sequence is split into shorter segments. In this case, the individual standardisation is much more important to minimise statistical bias during subsequent tests.

Figure $4 \mathrm{~b}$ illustrates the DFA2 band for 100 individually standardised lrc model series of spectral exponent $\beta=0.6$ (thin orange lines). Clearly, the black empirical curve fits much better into this band.

The thick red lines in Fig. 4 indicate the DFA2 curve for the tree ring proxy SST series (see Fig. 2). It covers a much longer time interval than the instrumental SST data (424 


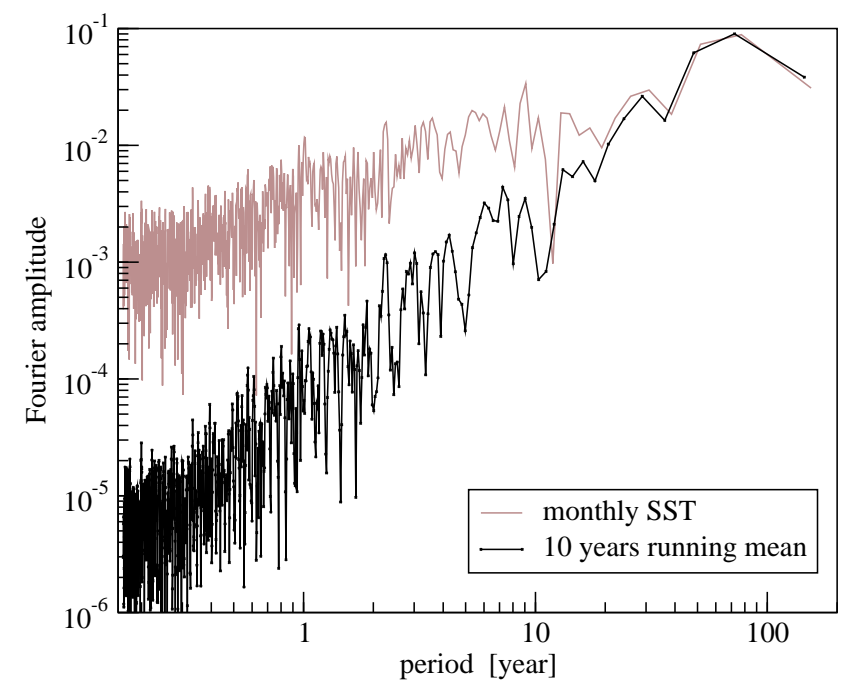

Fig. 6. Power spectra of the original monthly SST series (brown) and its 121 month running average, the AMO index (black with symbols), as a function of period length.

years in our analysis), however its temporal resolution of 1 year limits the statistical confidence of a similar modeling procedure presented for the monthly record. Therefore we performed the fitting-modelling-splitting test only for the instrumental monthly mean SST data. The DFA2 fluctuation function obviously has a lower magnitude for annual mean values compared to monthly fluctuations, and it is shifted on the horizontal axis as well. Still it is fully consistent with the DFA2 curve of the monthly SST series illustrated by the blue dashed lines in Fig. 4. It has the same slope, and enhances the difference between an $1 \mathrm{rc}$ and an AR(1) process with an asymptotic DFA slope of 1/2 (see Fig. 4a).

As it is already mentioned, Fourier spectra convey the same mathematical information as DFA $p$ correlation curves (Ghil et al., 2002), nevertheless Fig. 5 further illustrates the differences between the basic model assumptions. Various versions of Fig. 5a appeared in many papers with the conclusion that the small frequency peak at around $0.0012-$ 0.0017 month $^{-1}$ (according to a period of 50-70 years) is a statistically significant signature of a clean oscillatory mode. However, Fig. 5 b demonstrates that the peak is not significant for an lrc process, it is rather a finite size effect. (The power spectrum for the annual proxy SST series is not shown, it sinks into the plotted bands.)

\section{The effects of smoothing}

Thompson et al. (2010) convincingly pointed out that an undesirable side effect of high frequency filtering is the smearing out of jumpwise, abrupt changes, also in cases when the reason is some physical effect instead of noise. The definition of AMO index contains the filtering by a 10 -year running

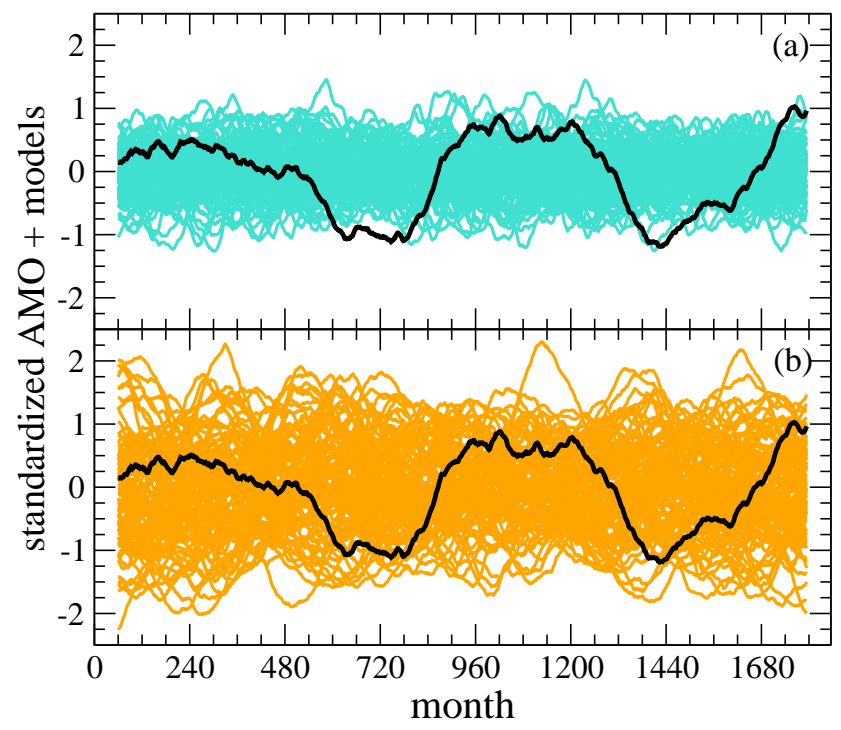

Fig. 7. Smoothed (121-point running mean) curves for (a) fitted $\mathrm{AR}(1)$, and (b) lrc model segments (thin coloured lines) after standardisation. The heavy black curve is the same for the standardised monthly AMO series (also shown in Fig. 1).

mean (see Figs. 1 and 2) which certainly helps to distinguish "warm" and "cold" intervals.

Figure 6 illustrates the effects of the 121-point running mean smoothing of the original monthly SST series. The interesting fact is that all spectral components of period $<30$ years are gradually suppressed, the highest frequencies are damped by more than two orders of magnitude. Meanwhile, the low frequency tail remains intact. Unfortunately this means only 3-4 points in the Fourier space as a consequence of limited record length. Note that the spectrum of AMO index (black curve in Fig. 6) retains its continuous powerlaw shape, the presence of pronounced isolated peaks is not salient.

Our final test aims to check the effect of smoothing by the running mean filter of the two model sets, the results are shown in Fig. 7. Note again that 121-point running means were determined on individually standardised model segments. The comparison of the cyan band in Fig. 7a with the AMO index suggests that the latter presents an extreme case if it is an $\mathrm{AR}(1)$ process, local minima and maxima coincide with the very edge of the model ensemble. However, when we assume that the AMO signal is an lrc process, the clean oscillation of a fixed frequency becomes an artifact of limited length and strong smoothing: Fig. 7b exhibits several model signals in the background (thin orange lines) with much larger amplitudes and similar length of "period". 


\section{Conclusions}

If we reject the hypothesis that the AMO signal represents a physical oscillatory mode of oceanic circulation, we should certainly comment on the opposite conclusions of the literature. First of all, note that the appearance of the same or very similar AMO-like patterns in other climate variables measured or reconstructed for the same 15-20 decades (Kushnir, 1994; Enfield et al., 2001; Goldenberg et al., 2001; Knight et al., 2006; Trenberth and Shea, 2006; Frankcombe et al., 2008; Thompson et al., 2010; Wang and Dong, 2010) does not prove the existence of a real oscillatory mode. It is widely accepted that large scale oceanic circulations are determining factors of the weather and climate, thus whatever changes SST over a significant geographic area, its effects will be appreciable in the whole coupled system.

The most popular explanation of the appearance of well defined oscillations in a noisy systems is based on mode selection. Indeed, model analysis by te Raa and Dijkstra (2002) and especially by Frankcombe et al. (2009) demonstrated that large scale oceanic circulation driven by differential heating and rotation produces SST oscillations. According to the results, both spatial and temporal correlations in the external noise were important for the excitation of possible multidecadal modes, with the amplitude of oscillation increasing with stronger temporal correlation. Note however that statistical significance of the filtered time series was tested by the red noise hypothesis (based on plots like Fig. 5a), thus the possible consequences of long range power-law correlations were not considered.

We repeat again, there is no doubt that low frequency SST variability is a prevailing feature of oceanic dynamics. Both DFA and Fourier spectral analysis suggest that the dominant time scales are indeed span over decades, because they have the strongest spectral magnitude. However both DFA and Fourier spectra exhibit a continuous power-law shape without the apparent presence of one (or more) well defined oscillation frequency. As we have demonstrated, filtering has a side effect of giving enhanced weights of low frequency components, and limited record lengths can easily lead to the impression of strict periodicity.

Acknowledgements. This work was supported by the Hungarian Science Foundation (OTKA) under Grant No. NK72037, the European Commissions RECONCILE-226365-FP7-ENV-2008-1 project, and by the European Union and the European Social Fund under the grant agreement no. TÁMOP 4.2.1./B-09/1/KMR-20100003 .

Edited by: W. Hsieh

Reviewed by: three anonymous referees

\section{References}

Ashkenazy, Y. and Tziperman, E.: Are the 41 kyr glacial oscillations a linear response to Milankovitch forcing?, Quat. Sci. Rev., 23, 1879-1890, doi:10.1016/j.quascirev.2004.04.008, 2004.

Cane, M. A. and Zebiak, S. E.: A theory for el nino and the southern oscillation, Science, 228, 1085-1087, 1985.

Delworth, T. L., Manabe, S., and Stouffer, R. J.: Interdecadal variations of the thermohaline circulation in a coupled oceanatmosphere model, J. Climate, 6, 1993-2011, 1993.

Ditlevsen, P. D., Kristensen, M. S., and Andersen, K. K.: The recurrence time of Dansgaard-Oeschger events and limits on the possible periodic component, J. Climate, 18, 2594-2603, doi:10.1175/JCLI3437.1, 2005.

Dong, B. and Sutton, R. T.: Mechanism of interdecadal thermohaline circulation variability in a coupled ocean-atmosphere GCM, J. Climate, 18, 1117-1135, 2005.

Enfield, D. B., Mestas-Nuñez, A. M., and Trimble, P. J.: The Atlantic Multidecadal Oscillation and its relation to rainfall and river flows in the continental U.S., Geophys. Res. Lett., 28, 2077-2080, doi:10.1029/2000GL012745, 2001.

Fox, C. G.: An inverse Fourier transform algorithm for generating random signals of a specified spectral form, Computers Geosci., 13, 369-374, doi:10.1016/0098-3004(87)90009-4, 1987.

Frankcombe, L. M. and Dijkstra, H. A.: Coherent multidecadal variability in North Atlantic sea level, Geophys. Res. Lett., 36, L15604, doi:10.1029/2009GL039455, 2009.

Frankcombe, L. M., Dijkstra, H. A., and von der Heydt, A.: Subsurface signatures of the Atlantic Multidecadal Oscillation, Geophys. Res. Lett., 35, L19602, doi:10.1029/2008GL034989, 2008.

Frankcombe, L. M., Dijkstra, H. A., and von der Heydt, A.: Noise induced multidecadal variability in the North Atlantic: Excitation of normal modes, J. Phys. Oceanogr., 39, 220-233, 2009.

Frankignoul, C. and Hasselmann, K.: Stochastic climate models, Part II, Application to sea-surface temperature anomalies and thermocline variability, Tellus, 29, 289, doi:10.1111/j.21533490.1977.tb00740.x, 1977.

Ganopolski, A. and Rahmstorf, S.: Abrupt glacial climate changes due to stochastic resonance, Phys. Rev. Lett., 88, 038501, doi:10.1103/PhysRevLett.88.038501, 2002.

Ghil, M., Allen, M. R., Dettinger, M. D., Ide, K., Kondrashov, D., Mann, M. E., Robertson, A. W., Saunders, A., Tian, Y., Varadi, F., and Yiou, P.: Advanced spectral methods for climatic time series, Rev. Geophys., 40, 1, doi:10.1029/2000RG000092, 2002.

Godfrey, C. M., Wilks, D. S., and Schultz, D. M.: Is the January Thaw a statistical phantom?, B. Am. Meteorol. Soc., 83, 53-62, doi:10.1175/1520-0477(2002)083<0053:ITJTAS > 2.3.CO;2, 2002.

Goldenberg, S. B., Landsea, C. W., Mestas-Nuñez, A. M., and Gray, W. M.: The recent increase in Atlantic hurricane activity: Causes and implications, Science, 293, 474-479. doi:10.1126/science.1060040, 2001.

Gray, S. T., Graumlich, L. J., Betancourt, J. L., and Pederson, G. T.: A tree-ring based reconstruction of the Atlantic Multidecadal Oscillation since 1567 A.D., Geophys. Res. Lett., 31, L12205. doi:10.1029/2004GL019932, 2004.

Heneghan, C. and McDarby, G.: Establishing the relation between detrended fluctuation analysis and power spectral density analysis for stochastic processes, Phys. Rev. E, 62, 6103-6110, doi:10.1103/PhysRevE.62.6103, 2000. 
Hurrell, J. W.: Decadal trends in the North Atlantic Oscillation: Regional temperatures and precipitation, Science, 269, 676-679, 1995.

Huybers, P. and Wunsch, C.: Obliquity pacing of the late Pleistocene glacial terminations, Nature, 434, 491-494, doi:10.1038/nature03401, 2005.

Jungclaus, J. H., Haak, H., Latif, M., and Mikolajewicz, U.: ArcticNorth Atlantic interactions and multidecadal variability of the meridional overturning circulation, J. Climate, 18, 4013-4031, 2005.

Kerr, R. A.: A North Atlantic climate pacemaker for the centuries, Science, 288, 1984-1986, doi:10.1126/science.288.5473.1984, 2000.

Király, A. and Jánosi, I. M.: Stochastic modeling of daily temperature fluctuations, Phys. Rev. E, 65, 051102, doi:10.1103/PhysRevE.65.051102, 2002.

Knight, J. R.: The Atlantic Multidecadal Oscillation inferred from the forced climate response in coupled general circulation models, J. Climate, 22, 1610-1625, doi:10.1175/2008JCLI2628.1, 2009.

Knight, J. R., Allan, R. J., Folland, C. K., Vellinga, M., and Mann, M. E.: A signature of persistent natural thermohaline circulation cycles in observed climate, Geophys. Res. Lett., 32, L20708, doi:10.1029/2005GL024233, 2005.

Knight, J. R., Folland, C. K., and Scaife, A. A.: Climate impacts of the Atlantic Multidecadal Oscillation, Geophys. Res. Lett., 33, L17706, doi:10.1029/2006GL026242, 2006.

Kushnir, Y.: Interdecadal variations in North Atlantic sea surface temperature and associated atmospheric conditions, J. Climate, 7, 141-157, 1994.

Monetti, R. A., Havlin, S., and Bunde, A.: Long-term persistence in the sea surface temperature fluctuations, Physica A, 320, 581589, doi:10.1016/S0378-4371(02)01662-X, 2003.
Ottera, O. H., Bentsen, M., Drange, H., and Suo, L.: External forcing as a metronome for Atlantic multidecadal variability, Nat. Geosci., 3, 688-694, doi:10.1038/NGEO955, 2010.

Park, W. and Latif, M.: Pacific and Atlantic multidecadal variability in the Kiel Climate Model, Geophys. Res. Lett., 37, L24702, doi:10.1029/2010GL045560, 2010.

Schlesinger, M. E. and Ramankutty, N.: An oscillation in the global climate system of period 65-70 years, Nature, 367, 723-726, doi:10.1038/367723a0, 1994.

Sutton, R. T. and Allen, M. R.: Decadal predictability of North Atlantic sea surface temperature and climate, Nature, 388, 563567, doi:10.1038/41523, 1997.

te Raa, L. A. and Dijkstra, H. A.: Instability of the thermohaline ocean circulation on interdecadal timescales, J. Phys. Oceanogr., 32, 138-160, 2002.

Thompson, D. W. J., Wallace, J. M., Kennedy, J. J., and Jones, P. D.: An abrupt drop in Northern Hemisphere sea surface temperature around 1970, Nature, 467, 444-447, doi:10.1038/nature09394, 2010.

Timmermann, A., Latif, M., Voss, R., and Grotzner, A.: Northern Hemisphere interdecadal variability: A coupled air-sea mode, J. Climate, 11, 1906-1931, 1998.

Trenberth, K. E.: Signal versus Noise in the Southern Oscillation, Mon. Weather Rev., 112, 326-332, 1984.

Trenberth, K. E. and Shea, D. J.: Atlantic hurricanes and natural variability in 2005, Geophys. Res. Lett., 33, L12704, doi:10.1029/2006GL026894, 2006.

von Storch, H. and Zwiers, F. W.: Statistical Analysis in Climate Research, Cambridge Univ. Press, Cambridge, 1999.

Wang, C. and Dong, S.: Is the basin-wide warming in the North Atlantic Ocean related to atmospheric carbon dioxide and global warming?,, Geophys. Res. Lett., 37, L08707, doi:10.1029/2010GL042743, 2010. 